\title{
Correlation dynamics during a slow interaction quench in a one-dimensional Bose gas
}

\author{
Jean-Sébastien Bernier, ${ }^{1}$ Roberta Citro, ${ }^{2}$ Corinna Kollath,${ }^{3}$ and Edmond Orignac ${ }^{4}$ \\ ${ }^{1}$ Department of Physics and Astronomy, University of British Columbia, Vancouver V6T 1Z1, Canada. \\ ${ }^{2}$ CNR-SPIN and Dipartimento di Fisica "E. R. Caianiello", Università degli Studi di Salerno, Via Giovanni Paolo II, I-84084 Fisciano, Italy. \\ ${ }^{3}$ HISKP, University of Bonn, Nussallee 14-16, D-53115 Bonn, Germany. \\ ${ }^{4}$ Laboratoire de Physique de l'École Normale Supérieure de Lyon, CNRS UMR5672, 46 Allée d'Italie, F-69364 Lyon Cedex 7, France.
}

(Dated: April 9, 2018)

\begin{abstract}
We investigate the response of a one-dimensional Bose gas to a slow increase of its interaction strength. We focus on the rich dynamics of equal-time single-particle correlations treating the Lieb-Liniger model within a bosonization approach and the Bose-Hubbard model using the time-dependent density-matrix renormalization group method. For short distances, correlations follow a power-law with distance with an exponent given by the adiabatic approximation. In contrast, for long distances, correlations decay algebraically with an exponent understood within the sudden quench approximation. This long distance regime is separated from an intermediate distance one by a generalized Lieb-Robinson criterion. At long times, in this intermediate regime, bosonization predicts that single-particle correlations decay following a stretched exponential. This latter regime is unconventional as, for one-dimensional interacting systems, the decay of single-particle correlations is usually algebraic within the Luttinger liquid picture. We develop here an intuitive understanding for the propagation of correlations, in terms of a generalized light-cone, applicable to a large variety of systems and quench forms.
\end{abstract}

PACS numbers: 67.85.-d, 03.75.Kk, 03.75.Lm, 67.25.D-

Introduction: Recent advances in the development of fast probing and control techniques applicable to correlated systems have opened up the possibility to dynamically prepare complex quantum many-body states. For example, effective phase transitions have been induced through the application of external driving fields [1-3] and states, such as a Bell state of ions or a Tonks-like state in a quantum gas, have been realized using tailored environments [4, 5]. In fact, the dynamical preparation of states promises to have an important impact in fields as diverse as condensed matter physics, quantum information, quantum optics and ultracold atomic physics. On the theoretical side, despite tremendous progress in recent years, many of the basic concepts behind the dynamical generation of states still remain to be understood.

In this article, we focus on the preparation of unconventional states in isolated systems using slow parameter changes. Considerable experimental efforts have been devoted to understand slow quench dynamics [6- $[10]$. However, in these works, as well as in many theoretical ones (see Ref. 11 and references therein), the emphasis has been put on understanding how energy is absorbed and defects produced.

In recent years, the focus has partially shifted towards the study of longer range correlation dynamics during a slow parameter quench [12-22]. Understanding the evolution of such correlations is paramount as the nature of many-body quantum states are typically characterized by longer range correlators. Interestingly, light-cone-like spreading [23, 24] of parity correlations, both in space and time, has even been observed experimentally in an interacting one-dimensional bosonic gas after a sudden quench of the optical lattice depth [25]. For slow quenches, a similar linear light-cone-like evolution of correlations has been predicted for density correlations in bosonic systems [20] and for single-particle correlations in fermionic systems [19].

We analyze here the correlation dynamics during a slow linear increase of the interaction strength, at zero temperature, in two paradigmatic one-dimensional interacting models: the Lieb-Liniger and Bose-Hubbard models. We show that a generalized Lieb-Robinson bound describes the evolution of single-particle correlations. This bound can be understood within a simple picture involving quasiparticle pairs created during the quench. At each instant in time, the quasiparticles propagate at their instantaneous velocity: as this velocity is time-dependent the evolution front possesses a non-trivial functional form. This non-trivial form contrasts with the linear evolution front of correlations, the horizon, which arises after a sudden quench and is due to a constant quasiparticle velocity [24]. The structure of the correlation front can be extracted solely from the knowledge of the quasiparticle velocity and does not require a detailed understanding of the more complicated correlation function. In fact, the approach developed here can be applied to various interacting systems.

For the one-dimensional models under study in this article, we find that outside the bound the single-particle correlations decay algebraically with distance with an exponent determined by the initial Luttinger parameter and decreased amplitude. In contrast, inside the bound, the correlations present much more interesting dynamics. For short distances, the algebraic decay depends on the ramp time [16, 19]. While for larger distances and quench times, the correlations, within the Lieb-Liniger model, decay following a stretched exponential. This particular decay form is unexpected as, even for instantaneous quenches, an algebraic decay persists at all distances and times [26]. A similar stretched exponential behavior was found in Ref. 16 (without a time-dependent prefactor). In the rest of the article, we analyze in detail the evolution of singleparticle correlations, and highlight the different regimes both in position and momentum space.

Model: Bosonic atoms in a one-dimensional wave guide 
can be described by the Lieb-Liniger (LL) model

$$
H=\int d x\left[-\frac{\hbar^{2}}{2 m} \psi^{\dagger}(x) \partial_{x}^{2} \psi(x)+\frac{g(t)}{2} \rho(x)^{2}\right]
$$

with $\psi(x)$ the boson annihilation operator and $\rho=$ $\psi(x)^{\dagger} \psi(x)$ the density. The interaction strength $g$ is related to the $s$-wave scattering length $a_{s}$ of the atoms and to the transverse trapping frequency $\omega_{\perp}$ by $g \approx 2 \pi \hbar \omega_{\perp} a_{s}$. We assume that the gas is initially prepared at a certain interaction strength $g(t)=g_{0}$ and that for $t>0$ a linear variation of the interaction strength of the form $g(t)=g_{0}+\left(g_{f}-g_{0}\right) \frac{t}{t_{f}}$ is performed. Experimentally this variation can be achieved, for example, by using a Feshbach resonance or by varying the intensity of the transverse trapping [27].

A similar interaction quench can be done by confining bosonic atoms to an optical lattice potential along the onedimensional direction. The theoretical model describing this situation is the Bose-Hubbard model given by

$$
\mathcal{H}=-J \sum_{l}\left(b_{l+1}^{\dagger} b_{l}+\text { h.c. }\right)+\frac{U(t)}{2} \sum_{l} \hat{n}_{l}\left(\hat{n}_{l}-1\right)
$$

with $b_{l}^{\dagger}$ the operator creating a boson at site $l$ and $\hat{n}_{l}=b_{l}^{\dagger} b_{l}$ the local density operator. The first term of the Hamiltonian corresponds to the kinetic energy of atoms with hopping amplitude $J$ while the second term is the potential energy with onsite interaction of strength $U$. Taking the continuum limit of the Bose-Hubbard model in the superfluid phase, this model can be mapped onto the LL Hamiltonian [28]. In this case, the linear interaction quench $g(t)$ translates into a linear change of the interaction amplitude $U(t)$.

For both models, in the superfluid phase, the low energy physics is well described by the Tomonaga-Luttinger liquid (TLL) Hamiltonian [29, 30]

$$
H=\sum_{q} \frac{q^{2}}{2 \pi}\left[u(t) K(t) \theta(q) \theta(-q)+\frac{u(t)}{K(t)} \phi(q) \phi(-q)\right]
$$

where $\phi(x)=\frac{1}{\sqrt{L}} \sum_{q} \phi(q) e^{i q x} e^{-|q| \alpha / 2}$ and $\theta(x)=$ $\frac{1}{\sqrt{L}} \sum_{q} \theta(q) e^{i q x} e^{-|q| \alpha / 2}$ are conjugate fields satisfying the canonical commutation relation $\left[\phi(x), \nabla \theta\left(x^{\prime}\right)\right]=i \pi \delta(x-$ $\left.x^{\prime}\right)$. We have set here $\hbar=1$ and $\alpha$ is a short distance cutoff. The sound velocity $u$ and the Luttinger parameter $K$ are related to the parameters of the original Hamiltonians. These parameters can, for example, be extracted from the Bethe Ansatz solution of Eq. (11) [31] or through numerical approaches for the Bose-Hubbard Hamiltonian [28, 32].

In the LL model, the Galilean invariance ensures that the product $u(t) K(t)$ remains unchanged upon varying the interaction parameters [33] and thus $u(t) K(t)=u_{0} K_{0}$. For small linear changes of these parameters, this translates, to first order in the variation, to a time-dependent ratio $\frac{u(t)}{K(t)} \approx \frac{u_{0}}{K_{0}}(1+$ $\left.\frac{t}{t_{0}}\right)$ with $t_{0}=\frac{\pi u_{0} t_{f}}{K_{0}\left(g_{f}-g_{0}\right)}$ and a typical lengthscale $l_{0}=u_{0} t_{0}$. This result is then used to obtain expressions for the timedependent sound velocity $u(t) \approx u_{0} \sqrt{1+\frac{t}{t_{0}}}$ and for the time-dependent Luttinger parameter $K(t) \approx K_{0} / \sqrt{1+\frac{t}{t_{0}}}$. These expressions are still valid for small parameter variations in the Bose-Hubbard model given the relation $U_{0 / f} a=g_{0 / f}$ where $a$ is the lattice constant.

A major distinctive feature of the TTL model is that its low energy excitations are collective modes (density fluctuations) instead of individual quasiparticles. Hence, only quasi-long range order persists even down to zero temperature. This situation is exemplified by the anomalous (non-integer) powerlaw dependence of its correlation functions [29]. Moreover, the time-dependence does not introduce couplings between the different momentum modes of the TTL Hamiltonian. This leads to momentum decoupled equations of motion for the Fourier components of the fields of the form [16, 19, 20]

$$
\frac{d}{d t} \phi(q)=u_{0} K_{0} q \theta(q) \text { and } \frac{d}{d t} \theta(q)=-\frac{u(t)}{K(t)} q \phi(q) .
$$

The solutions for these equations of motion can be written using bosonic quasiparticles with creation and annihilation operators $a^{\dagger}$ and $a$ which diagonalize the Hamiltonian at $t=0$ :

$$
\begin{aligned}
& \phi(q, t)=2 \sqrt{\pi K_{0}}|q|\left[a_{q} F^{*}+a_{-q}^{\dagger} F\right], \\
& \theta(q, t)=\frac{1}{u(t) K(t) q} \sqrt{\frac{\pi K_{0}}{2|q|}}\left[a_{q} \frac{d}{d t} F^{*}+a_{-q}^{\dagger} \frac{d}{d t} F\right]
\end{aligned}
$$

where $F(q, t)$ is the solution of the equation

$$
\left(\frac{1}{u_{0} K_{0}} \frac{d^{2}}{d t^{2}} F(q, t)\right)=-\frac{u(t)}{K(t)} q^{2} F(q, t)
$$

with initial conditions $F(q, 0)=1,\left.\frac{d}{d t} F(q, t)\right|_{t=0}=i u_{0}|q|$. This solution can be expressed in terms of Bessel functions (see Eq. (9.1.51) of Ref. 34):

$$
\begin{aligned}
F(q, t)= & \frac{\pi s \tau^{\frac{1}{2}}}{\sqrt{3}}\left[J_{\frac{2}{3}}(s) J_{\frac{1}{3}}\left(s \tau^{\frac{3}{2}}\right)+J_{-\frac{2}{3}}(s) J_{-\frac{1}{3}}\left(s \tau^{\frac{3}{2}}\right)\right. \\
& \left.+i\left(J_{-\frac{1}{3}}(s) J_{\frac{1}{3}}\left(s \tau^{\frac{3}{2}}\right)-J_{\frac{1}{3}}(s) J_{-\frac{1}{3}}\left(s \tau^{\frac{3}{2}}\right)\right)\right]
\end{aligned}
$$

where $s\left(q, t_{0}\right)=\frac{2}{3} l_{0}|q|$ and $\tau\left(t, t_{0}\right)=1+\frac{t}{t_{0}}$ are the dimensionless momentum and time, respectively [35].

Evolution of the single-particle correlation function: In the following, we survey the rich behavior of the equal-time single-particle correlation function $G(x, t)=$ $\frac{1}{2}\left\langle\psi(x, t) \psi^{\dagger}(0, t)+h . c.\right\rangle$ during a slow interaction quench.

In the bosonization representation, the equal-time singleparticle correlation function takes the form

$$
\begin{aligned}
G(x, t)_{q \simeq 0} & =A_{0}^{2}\left\langle e^{i \theta(x, t)} e^{-i \theta(0, t)}\right\rangle \\
& =A_{0}^{2} e^{-\frac{1}{2} I(\xi, \tau, \tilde{\alpha})}
\end{aligned}
$$

where $A_{0}$ is a non-universal constant which depends on the underlying microscopic model. We introduced for convenience the dimensionless length $\xi=\frac{3 x}{2 l_{0}}$ and, correspondingly, the dimensionless short distance cut-off $\tilde{\alpha}=\frac{3 \alpha}{2 l_{0}}$. The 
function $I(\xi, \tau, \tilde{\alpha})$ of Eq. (7) is then given by

$$
\begin{aligned}
I(\xi, \tau, \tilde{\alpha})= & \frac{\pi^{2} \tau^{2}}{3 K_{0}} \int_{0}^{\infty} d s s e^{-\tilde{\alpha} s}(1-\cos s \xi) \\
\times & {\left[\left(J_{\frac{2}{3}}(s) J_{-\frac{2}{3}}\left(s \tau^{\frac{3}{2}}\right)-J_{-\frac{2}{3}}(s) J_{\frac{2}{3}}\left(s \tau^{\frac{3}{2}}\right)\right)^{2}+\right.} \\
& \left.\left(J_{-\frac{1}{3}}(s) J_{-\frac{2}{3}}\left(s \tau^{\frac{3}{2}}\right)+J_{\frac{1}{3}}(s) J_{\frac{2}{3}}\left(s \tau^{\frac{3}{2}}\right)\right)^{2}\right] .
\end{aligned}
$$

From the equation above it immediately follows that Eq. (7) only depends on the dimensionless variables $\tau, \xi, \tilde{\alpha}$ and not separately on $t, t_{0}, x$ and $\alpha$. This implies that, for a given final value of the interaction strength, increasing the ramp velocity, $\frac{1}{t_{0}}$, mainly enters the expressions through an increased rescaled length $\xi$.

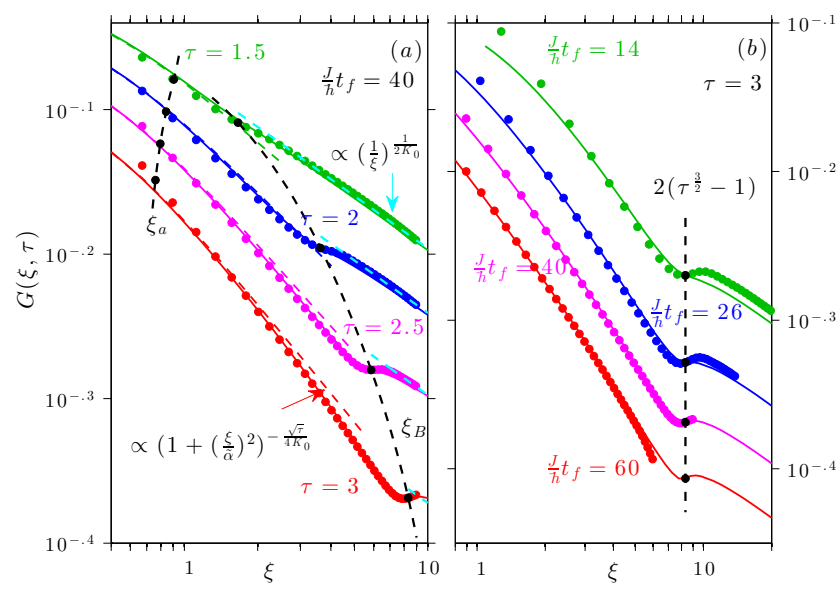

FIG. 1: Decay of single-particle correlations with increasing distance for different $\tau$ and $t_{f}$. Comparison between results obtained using bosonization Eq. (7) with Luttinger liquid parameters $K_{0}=4.1561$ and $u_{0}=1.3323$ (solid lines) and using timedependent density-matrix renormalization group (t-DMRG) for the Bose-Hubbard model (circles) for a quench from $U_{0}=J$ (lattice length: $L=100$, filling: $n=1$, maximum number of bosons per site: 6$)$. (a) Time evolution for different values of $\tau$ and for a fixed value of $t_{f}=40 \frac{\hbar}{J}$. The two dashed lines intersecting all $\tau$ data sets are the bounds: (left) $\xi_{a}=\tau^{-1 / 4}$ and (right) $\xi_{B}=2\left(\tau^{3 / 2}-1\right)$. The colored dashed lines on the left of $\xi_{B}$ are curves proportional to the function $\left(1+\left(\frac{\xi}{\tilde{\alpha}}\right)^{2}\right)^{-1 /(4 K(\tau))}$; while the dashed lines on the right of $\xi_{B}$ are curves proportional to the function $\xi^{-1 /\left(2 K_{0}\right)}$. (b) Comparison between different ramp times $t_{f}$ for a fixed value of $\tau=3$. The vertical dashed line is the bound $\xi_{B}=2\left(\tau^{3 / 2}-1\right)$.

Asymptotic expansion of the single-particle correlation function: The time evolution of single-particle correlations described by Eqs. (7) and (8) is extremely rich. Typical time evolutions of these correlations with distance are shown in panel $(a)$ of Fig. 1 for both the Bose-Hubbard model and the bosonization approach. For the chosen parameters, we found very good agreement between the two evolutions at longer distances, as long as an additional time-dependent prefactor is multiplied to the expression obtained using bosonization.
This prefactor corrects for the short distance behavior which is not properly taken into account by the low energy theory. As expected, the bosonization description works best for slow and small parameter changes. In particular, deviations are observed when the Mott-insulating phase of the Bose-Hubbard model is approached or when too many excitations are created.

Initially, before the slow quench begins (at $\tau=1$ within our formalism), the correlation function decays algebraically with distance as $G(\xi)=A_{0}^{2}\left(1+(\xi / \tilde{\alpha})^{2}\right)^{-1 /\left(4 K_{0}\right)}$. This behavior is typical of a Luttinger liquid. Then, as the interaction strength is slowly ramped up, the form of the correlation function evolves. For small $\xi$ and sufficiently short $\tau$, changes are minimal as the correlation function still decays algebraically, but the exponent is now determined by the timedependent Luttinger parameter $K(t)=K_{0} / \sqrt{\tau}$ showing up in the exponent [36]. This result implies that for short dimensionless distances, $\xi_{a}:=\tau^{-1 / 4} \gg \xi$, the correlations react instantaneously to the slow interaction change and adjust to the ground state decay corresponding to the current interaction value (see panel $(a)$ of Fig. 1). The main contribution to this mechanism comes from quasiparticles with large momenta $q \gg \frac{1}{l_{0}}$. This adiabatic regime spatially decreases with time and disappears completely when $\xi_{a}(t) \approx \tilde{\alpha}$, where $\tilde{\alpha}$ is the dimensionless short distance cut-off.

For larger distances, the correlations deviate much more from their standard initial form and a dip appears. The formation of this dip is a clear signal of the non-equilibrium nature of the physics at play. For distances beyond this dip, the initial algebraic decay, $\xi^{-1 /\left(2 K_{0}\right)}$, reappears as one can see in panel $(a)$ of Fig. 1 The position of the dip coincides approximately with the correlation evolution front. The time-dependent position of this front can be understood by considering the propagation of quasiparticles. At any given time $t$, the system Hamiltonian is diagonal in its instantaneous quasiparticles as $H\left(t^{\prime}\right)=\sum_{q} u\left(t^{\prime}\right)|q| a_{q}^{\dagger}\left(t^{\prime}\right) a_{q}\left(t^{\prime}\right)+\frac{1}{2}$. Assuming discrete time steps, this means that the action of the Hamiltonian at time $t-\delta t$, diagonal in its own quasiparticles, has created (and annihilated) entangled quasiparticle pairs $a_{q}^{\dagger}(t) a_{-q}^{\dagger}(t)$. These entangled quasiparticles, forming a pair, propagate with velocity $u(t)$ in opposite direction and thereby carry correlations over a distance $2 u(t) d t$ within a time interval $d t$. Hence, for points separated by a distance $\xi$ larger than $\xi_{B}=\frac{3}{l_{0}} \int_{0}^{t} d t^{\prime} u\left(t^{\prime}\right)$, the single-particle correlation decay is unaffected by the change in the interaction aside from an overall prefactor. For the system under study, $u(t)=u_{0} \sqrt{1+\frac{t}{t_{0}}}$ and we find that $\xi_{B}=2\left(\tau^{3 / 2}-1\right)$. Thus, the evolution front beyond which correlations still follow the initial algebraic decay is given by $\xi_{B}$ as evidenced in Fig. 1. In particular, the position of the bound does not depend on the ramp velocity and time separately as can be seen in panel (b) of Fig 1. One clearly sees from there that, for a given $\tau$, the position $\xi$ of the dip (measured in units of $l_{0}$ ) is the same for different ramp times. The existence of such a propagation front is reminiscent of the light-cone-like evolution of corre- 
lations recently investigated in the context of instantaneous quenches [23-25, 37-39].

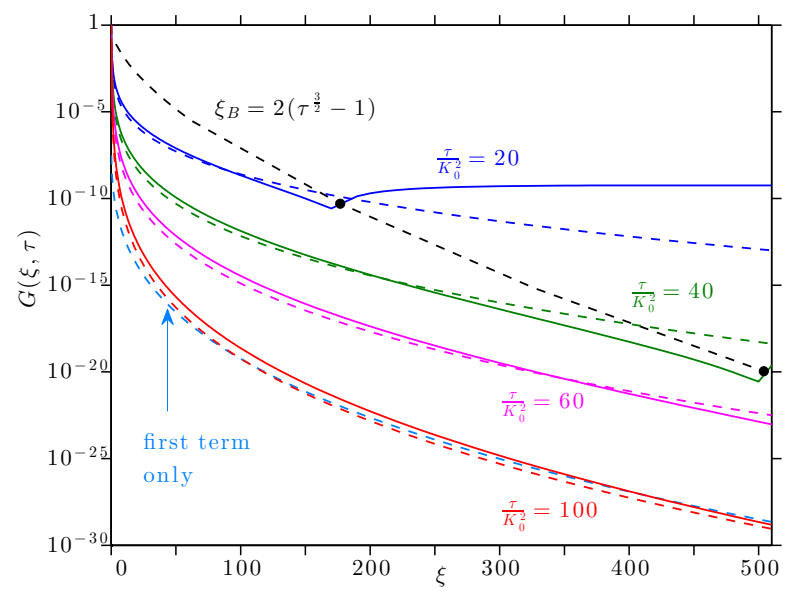

FIG. 2: Behavior of single-particle correlations with increasing distance for large values of $\tau / K_{0}^{2}$. Exact evaluation of the bosonization expression, Eq. (8) - solid lines, is compared to the full approximate expression, Eq. (9) - dashed lines. For $\tau / K_{0}^{2}=100$, we also compare the exact expression to the first exponential term of Eq. 9. In the large $\tau$ limit, if one adjusts the prefactor correctly, the stretched exponential provides a good description of the correlation decay before $\xi_{B}$. The black dashed line indicates the position of the evolution front $\xi_{B}=2\left(\tau^{3 / 2}-1\right)$. Used parameters: $s_{\min }=10^{-5}, s_{\max }=60$ (the lower and upper cut-offs in Eq. (8)) and $\tilde{\alpha}=0.1$.

For larger dimensionless times, as illustrated in Fig. 2, an additional decay regime takes place at intermediate distances before the bound $\xi_{B}$. This interesting behavior shows up in the bosonization approach and takes the form

$$
\begin{aligned}
& G(\xi, \tau)_{q \simeq 0} \simeq \tilde{\mathcal{C}}(\tau) \times \\
& \quad \exp \left(-\frac{2^{\frac{1}{3}} \pi^{2} \tau^{\frac{1}{2}}}{K_{0} \Gamma\left(\frac{1}{3}\right)^{3}} \xi^{\frac{1}{3}}\right) \exp \left(\frac{\pi^{\frac{3}{2}} \tau^{\frac{1}{2}} \Gamma\left(\frac{1}{6}\right)}{6 K_{0} \Gamma\left(\frac{1}{3}\right) \Gamma\left(\frac{2}{3}\right)^{2}} \frac{1}{\xi^{\frac{1}{3}}}\right)
\end{aligned}
$$

with $\tilde{\mathcal{C}}(\tau)$ a prefactor independent of $\xi$. For intermediate $\tau$, both exponential terms are required to adequately reproduce the behavior of Eq. (8) as shown in Fig. 2. However, for values of $\tau$ whose corresponding bound $\xi_{B}$ is located at sufficient large $\xi$, only the first exponential term is important. In this case single-particle correlations decay with distance as a stretched exponential, a similar decay was found in Ref. 16. Such a functional form is unconventional for Luttinger liquids as, typically, correlations decay algebraically in these systems. Even for sudden interaction quenches in both bosonic and fermionic systems [26, 40] and for slow quenches in fermionic systems [19], only algebraic decay of correlations have been uncovered. The presence of such an unusual functional form is mainly due to the reinforcement of the amplitude of phase fluctuations at low momenta with respect to the equilibrium case. The quench generates an unusual (non-thermal) distribution of quasiparticles around $\frac{3}{2 \xi_{B} l_{0}}<q \leq \frac{1}{l_{0}}$ [36].
Moreover, as the appearance of the stretched exponential decay is limited to large values of $\tau$, this regime only occurs for relatively large parameter changes $t \gg t_{0}$. It is still an open question, whether this stretched exponential decay regime arises within the Bose-Hubbard model. As this regime only occurs for large parameter changes, the TLL model might not describe properly the dynamics of the BoseHubbard model and relaxation mechanisms not present in the TLL model might dominate the evolution. A careful analysis of this last point would be extremely valuable but is left to further studies.

Experimental implementation and detection: Onedimensional interacting bosonic gases have been realized experimentally using various setups [41-43]. The timedependence of the ratio of potential to kinetic energy can be implemented using Fesbach resonances, or by varying the optical lattice depth or the transverse trapping.

Detection of the single-particle correlation function can also be carried out experimentally. Using radio-frequency pulses, atoms can be outcoupled from the one-dimensional Bose gas at two spatially separated positions and their interference is then observed after a free fall. This technique was successfully employed to measure the build-up of equaltime single-particle correlations in a Bose-Einstein condensate after a sudden decrease of its temperature [44, 45]. Another possible detection scheme relies on time-of-flight (TOF) measurements which provide, in the far-field limit, access to the momentum distribution $n(q)=\int d x e^{i q x} G(x)$. The very long distance behavior of the single-particle correlation is dominated by the Luttinger liquid power-law; however, at a critical wavevector, $q_{c}$, determined by the ballistic expansion condition, a crossover occurs and $n(q)$ is dominated by the Fourier transform of the stretched exponential. Therefore, at $q_{c} \sim m_{B} \xi_{B} l_{0} / t$ (with $m_{B}$ the atom mass) a crossover should be visible in the TOF measurements. One of the main challenges towards the observation of the evolution of correlations will be the realization of a relatively homogeneous gas as inhomogeneities can cause mass transport and mask the internal evolution [22, 46]. However, due to recent experimental advances [9, 47], we believe that, in the future, creating approximately box-shaped one-dimensional gases will be possible.

Conclusion: We uncovered various interesting regimes in the dynamics of single-particle correlations arising during the slow interaction quench of a one-dimensional Bose gas. We proposed a generalized picture for the propagation of the correlation evolution front based on the counterpropagation of entangled quasiparticle pairs moving at each point of time at their instanteneous velocity. Therefore, the evolution front does not simply spread as a light-cone as found following a sudden parameter change [26, 48], but aquires a more complex functional form. We expect this picture to apply to other models and quench forms as the evolution front can be predicted from the sole knowledge of the quasiparticle velocity. For example, we expect that for of a linear decrease of the interaction strength $U(t)=U_{0}\left(1-\frac{t}{t_{0}}\right)$, starting from a Mott-insulator, the propagation front will 
be of the form $4 J(2 n+1) t\left(1-\frac{8 n(n+1) J^{2}}{(2 n+1)^{2} U_{0}^{2}\left(1-t / t_{0}\right)}\right)$ as the maximal velocity of quasiparticles is given by $v_{\max } \approx$ $2 J(2 n+1)\left(1-\frac{8 n(n+1) J^{2}}{(2 n+1)^{2} U_{0}^{2}\left(1-t / t_{0}\right)^{2}}\right)$ where $n$ is the average filling [48]. These results may serve as a basis for comparison with experimental studies of unconventional time evolutions in many-body one-dimensional systems.

We are grateful to T. Giamarchi for helpful discussions and to G. Roux for his insights on related works. We acknowledge support from ANR (FAMOUS), SNFS under MaNEP and Divison II, NSERC of Canada and CIFAR.

[1] H. Lignier, C. Sias, D. Ciampini, Y. Singh, A. Zenesini, O. Morsch, and E. Arimondo, Phys. Rev. Lett. 99, 220403 (2007).

[2] D. N. Basov, R. D. Averitt, D. van der Marel, M. Dressel, and K. Haule, Rev. Mod. Phys. 83, 471 (2011).

[3] J. Struck, C. lschlger, R. Le Targat, P. Soltan-Panahi, A. Eckardt, M. Lewenstein, P. Windpassinger, and K. Sengstock, Science 333, 996 (2011).

[4] J. T. Barreiro, M. Mller, P. Schindler, D. Nigg, T. Monz, M. Chwalla, M. Hennrich, C. F. Roos, P. Zoller, and R. Blatt, Nature 470, 486 (2010).

[5] N. Syassen, D. M. Bauer, M. Lettner, T. Volz, D. Dietze, J. J. Garcia-Ripoll, J. I. Cirac, G. Rempe, and S. Dürr, Science 320, 1329 (2008).

[6] M. Greiner, O. Mandel, T. W. Hänsch, and I. Bloch, Nature (London) 419, 51 (2002).

[7] C.-L. Hung, X. Zhang, N. Gemelke, and C. Chin, Phys. Rev. Lett. 104, 160403 (2010).

[8] J. F. Sherson, C. Weitenberg, M. Endres, M. Cheneau, I. Bloch, and S. Kuhr, Nature (London) 467, 68 (2010).

[9] W. S. Bakr, A. Peng, M. E. Tai, R. Ma, J. Simon, J. I. Gillen, S. Fölling, L. Pollet, and M. Greiner, Science 329, 547 (2010).

[10] D. Chen, M. White, C. Borries, and B. DeMarco, Phys. Rev. Lett. 106, 235304 (2011).

[11] A. Polkovnikov, K. Sengupta, A. Silva, and M. Vengalattore, Rev. Mod. Phys. 83, 863 (2011).

[12] R. Schützhold, M. Uhlmann, Y. Xu, and U. R. Fischer, Phys. Rev. Lett. 97, 200601 (2006).

[13] F. M. Cucchietti, B. Damski, J. Dziarmaga, and W. H. Zurek, Phys. Rev. A 75, 023603 (2007).

[14] L. Cincio, J. Dziarmaga, M. M. Rams, and W. H. Zurek, Phys. Rev. A 75, 052321 (2007).

[15] R. W. Cherng and L. S. Levitov, Phys. Rev. A 73, 043614 (2006).

[16] A. Polkovnikov and V. Gritsev, Nature Physics 4, 477 (2008).

[17] M. Eckstein and M. Kollar, New Journal of Physics 12, 055012 (2010).

[18] M. Moeckel and S. Kehrein, New Journal of Physics 12, 055016 (2010).

[19] B. Dóra, M. Haque, and G. Zaránd, Phys. Rev. Lett. 106, 156406 (2011).

[20] J. Dziarmaga and M. Tylutki, Phys. Rev. B 84, 214522 (2011).

[21] D. Poletti and C. Kollath, Phys. Rev. A 84, 013615 (2011).

[22] J.-S. Bernier, D. Poletti, P. Barmettler, G. Roux, and C. Kollath, Phys. Rev. A 85, 033641 (2012).

[23] E. H. Lieb and D. W. Robinson, Communications in Mathematical Physics 28, 251 (1972).
[24] P. Calabrese and J. Cardy, Phys. Rev. Lett. 96, 136801 (2006).

[25] M. Cheneau, P. Barmettler, D. Poletti, M. Endres, P. Schauß, T. Fukuhara, C. Gross, I. Bloch, C. Kollath, and S. Kuhr, Nature 481, 484 (2012).

[26] M. A. Cazalilla, Phys. Rev. Lett. 97, 156403 (2006).

[27] I. Bloch, J. Dalibard, and W. Zwerger, Rev. Mod. Phys. 80, 885 (2008).

[28] C. Kollath, U. Schollwöck, J. von Delft, and W. Zwerger, Phys. Rev. A 69, 031601 (2004).

[29] T. Giamarchi, Quantum Physics in One Dimension (Oxford University Press, Oxford, 2004).

[30] M. A. Cazalilla, R. Citro, T. Giamarchi, E. Orignac, and M. Rigol, Rev. Mod. Phys. 83, 1405 (2011).

[31] E. H. Lieb and W. Liniger, Phys. Rev. 130, 1605 (1963).

[32] T. D. Kühner and H. Monien, Phys. Rev. B 58, R14741 (1998).

[33] F. D. M. Haldane, Phys. Rev. Lett. 47, 1840 (1981).

[34] M. Abramowitz and I. Stegun, eds., Handbook of mathematical functions (Dover, New York, 1972).

[35] Equivalently, the solution can be expressed using Airy functions, but the Bessel function representation leads to a more straightforward derivation of asymptotics.

[36] J.-S. Bernier, R. Citro, C. Kollath, and E. Orignac, Supplementary material for Correlation dynamics during a slow interaction quench in a one-dimensional Bose gas, attached to this manuscript (2013).

[37] S. Bravyi, M. B. Hastings, and F. Verstraete, Phys. Rev. Lett. 97, 050401 (2006).

[38] A. M. Läuchli and C. Kollath, J. Stat. Mech.: Theor. Exp. 5, 18 (2008).

[39] A. Mitra, Phys. Rev. B 87, 205109 (2013).

[40] C. Karrasch, J. Rentrop, D. Schuricht, and V. Meden, Phys. Rev. Lett. 109, 126406 (2012).

[41] T. Stöferle, H. Moritz, C. Schori, M. Köhl, and T. Esslinger, Phys. Rev. Lett. 92, 130403 (2004).

[42] B. Paredes, A. Widera, V. Murg, O. Mandel, S. Fölling, I. Cirac, G. V. Shlyapnikov, T. W. Hänsch, and I. Bloch, Nature 429, 277 (2004).

[43] T. Kinoshita, T. Wenger, and D. S. Weiss, Science 305, 1125 (2004).

[44] S. Ritter, A. Öttl, T. Donner, T. Bourdel, M. Köhl, and T. Esslinger, Phys. Rev. Lett. 98, 090402 (2007).

[45] T. Donner, S. Ritter, T. Bourdel, A. Öttl, M. Köhl, and T. Esslinger, Science 315, 1556 (2007).

[46] S. S. Natu, K. R. A. Hazzard, and E. J. Mueller, Phys. Rev. Lett. 106, 125301 (2011).

[47] A. L. Gaunt, T. F. Schmidutz, I. Gotlibovych, R. P. Smith, and Z. Hadzibabic, Phys. Rev. Lett. 110, 200406 (2013).

[48] P. Barmettler, D. Poletti, M. Cheneau, and C. Kollath, Phys. Rev. A 85, 053625 (2012). 


\section{Supplementary material}

\section{DERIVATION OF EQ. (8) OF THE MAIN TEXT}

Within the bosonization formalism the equal-time single-particle correlation function is

$$
\begin{aligned}
G(x, t)_{q \simeq 0} & =A_{0}^{2}\left\langle e^{i \theta(x, t)} e^{-i \theta(0, t)}\right\rangle \\
& =A_{0}^{2} e^{-\frac{1}{2}\left\langle(\theta(x, t)-\theta(0, t))^{2}\right\rangle} .
\end{aligned}
$$

The correlator appearing above is obtained from Eqs. (4) and (5) of the main text and is given by

$$
\left\langle\left(\theta(x, t)-\theta(0, t)^{2}\right\rangle=\frac{1}{2 K_{0} u_{0}^{2}} \int_{-\infty}^{+\infty} \frac{d q}{|q|^{3}} e^{-\alpha|q|}\left|\frac{d}{d t} F(q, t)\right|^{2}[1-\cos (q x)]\right.
$$

where the derivative of Eq. (6) of the main text is

$$
\frac{d}{d t} F(q, t)=\frac{\sqrt{3}}{2} \frac{u_{0}}{l_{0}} \pi \tau s^{2}\left[J_{\frac{2}{3}}(s) J_{-\frac{2}{3}}\left(s \tau^{\frac{3}{2}}\right)-J_{-\frac{2}{3}}(s) J_{\frac{2}{3}}\left(s \tau^{\frac{3}{2}}\right)+i\left(J_{-\frac{1}{3}}(s) J_{-\frac{2}{3}}\left(s \tau^{\frac{3}{2}}\right)+J_{\frac{1}{3}}(s) J_{\frac{2}{3}}\left(s \tau^{\frac{3}{2}}\right)\right)\right]
$$

with $J_{n}(y)$ the Bessel function of the first kind. Recall that $l_{0}=u_{0} t_{0}$ is the typical lengthscale, $s\left(q, t_{0}\right)=\frac{2}{3} l_{0}|q|$ the dimensionless momentum, and $\tau\left(t, t_{0}\right)=1+\frac{t}{t_{0}}$ the dimensionless time.

\section{ASYMPTOTIC EXPANSIONS}

In order to obtain analytical approximations for the single-particle function $G(x, t)_{q \simeq 0}=A_{0}^{2} e^{-\frac{1}{2} I(\xi, \tau, \tilde{\alpha})}$, we need to approximate the integral $I(\xi, \tau, \tilde{\alpha})$. We identify three important regimes: $s \ll \frac{2}{3} \tau^{-\frac{3}{2}}, \frac{2}{3} \tau^{-\frac{3}{2}} \ll s \ll \frac{2}{3}$ and $s \gg \frac{2}{3}$ (note that $\tau \geq 1$ and that we separate the regimes in $s$ at $\frac{2}{3}$ instead of 1 as this corresponds to a splitting at $\frac{1}{l_{0}}$ in momentum space). These three regimes determine the size of the arguments of the Bessel functions entering the integral $I(\xi, \tau, \tilde{\alpha})$. Therefore, we split this integral into three parts $I(\xi, \tau, \tilde{\alpha})=\mathfrak{G}(\xi, \tau)+\mathfrak{H}(\xi, \tau)+\mathfrak{Q}(\xi, \tau)$ with $\mathfrak{G}(\xi, \tau)=I\left(\xi, \tau, \tilde{\alpha}, 0, \frac{2}{3} \tau^{-\frac{3}{2}}\right), \mathfrak{H}(\xi, \tau)=I\left(\xi, \tau, \tilde{\alpha}, \frac{2}{3} \tau^{-\frac{3}{2}}, \frac{2}{3}\right)$, and $\mathfrak{Q}(\xi, \tau)=I\left(\xi, \tau, \tilde{\alpha}, \frac{2}{3}, \infty\right)$ using

$$
\begin{aligned}
I\left(\xi, \tau, \tilde{\alpha}, c_{1}, c_{2}\right)= & \frac{\pi^{2} \tau^{2}}{3 K_{0}} \int_{c_{1}}^{c_{2}} d s s e^{-\tilde{\alpha} s}[1-\cos (s \xi)] \times \\
& {\left[\left(J_{\frac{2}{3}}(s) J_{-\frac{2}{3}}\left(s \tau^{\frac{3}{2}}\right)-J_{-\frac{2}{3}}(s) J_{\frac{2}{3}}\left(s \tau^{\frac{3}{2}}\right)\right)^{2}+\left(J_{-\frac{1}{3}}(s) J_{-\frac{2}{3}}\left(s \tau^{\frac{3}{2}}\right)+J_{\frac{1}{3}}(s) J_{\frac{2}{3}}\left(s \tau^{\frac{3}{2}}\right)\right)^{2}\right] . }
\end{aligned}
$$

The Bessel functions of the first kind can take simpler approximated forms (using expressions taken from Ref. 1) in the two following regimes:

$$
\begin{aligned}
& \text { for } s \ll \frac{2}{3}, J_{\nu}(s) \sim\left(\frac{s}{2}\right)^{\nu} \frac{1}{\Gamma(\nu+1)} \\
& \text { for } s \gg \frac{2}{3}, J_{\nu}(s) \sim \sqrt{\frac{2}{\pi s}} \cos \left[s-\frac{\pi}{4}(2 \nu+1)\right] .
\end{aligned}
$$

\section{Large momentum contribution}

For the function $\mathfrak{Q}(\xi, \tau)$ which covers the regime $s \gg \frac{2}{3}$, we get

$$
\mathfrak{Q}(\xi, \tau) \simeq \frac{1}{K(\tau)} \int_{2 / 3}^{+\infty} \frac{d s}{s}[1-\cos (s \xi)] e^{-s \tilde{\alpha}}=\frac{1}{K(\tau)}\left\{E_{1}\left(\frac{2}{3} \tilde{\alpha}\right)-\frac{1}{2}\left[E_{1}\left(\frac{2}{3}(\tilde{\alpha}+i \xi)\right)+E_{1}\left(\frac{2}{3}(\tilde{\alpha}-i \xi)\right)\right]\right\}
$$

where $K(\tau)=\frac{K_{0}}{\sqrt{\tau}}$ and $E_{1}$ is the exponential integral function [1]. For $\xi \ll 1$, we find

$$
\mathfrak{Q}(\xi, \tau) \simeq \frac{1}{2 K(\tau)} \ln \left(1+\frac{\xi^{2}}{\tilde{\alpha}^{2}}\right)
$$


while for $\xi \gg 1$,

$$
\mathfrak{Q}(\xi, \tau) \simeq \frac{1}{K(\tau)}\left[\ln \left(\frac{e^{-\gamma_{E}}}{\frac{2}{3} \tilde{\alpha}}\right)+\frac{1}{\xi} \sin (\xi)+O\left(\xi^{-2}\right)\right]
$$

where $\gamma_{E}$ is Euler's constant.

\section{Small momentum contribution}

For the small momentum contribution, given by $\mathfrak{G}(\xi, \tau)$, we obtain the following expression

$$
\begin{aligned}
\mathfrak{G}(\xi, \tau) & \simeq \frac{1}{K_{0}} \int_{0}^{\frac{2 \xi}{3} \tau^{-\frac{3}{2}}} \frac{d u}{u}[1-\cos u] \\
& \simeq \frac{1}{K_{0}} \operatorname{Cin}\left(\frac{2 \xi}{3} \frac{1}{\tau^{\frac{3}{2}}}\right) .
\end{aligned}
$$

\section{Intermediate momentum contribution}

The most interesting contribution is the intermediate momentum one which is due to $\mathfrak{H}(\xi, \tau)$. This expression simplifies to

$$
\mathfrak{H}(\xi, \tau) \simeq \frac{2^{\frac{7}{3}} \pi \sqrt{\tau}}{3 K_{0}\left(\Gamma\left(\frac{1}{3}\right)\right)^{2}} \Phi_{1}\left(\xi, \tau^{\frac{3}{2}}\right)+\frac{2^{\frac{5}{3}} \pi \sqrt{\tau}}{3 K_{0}\left(\Gamma\left(\frac{2}{3}\right)\right)^{2}} \Phi_{2}\left(\xi, \tau^{\frac{3}{2}}\right)
$$

where

$$
\begin{aligned}
& \Phi_{1}\left(\xi, \tau^{\frac{3}{2}}\right)=\int_{\frac{2}{3} \tau^{-\frac{3}{2}}}^{\frac{2}{3}} \frac{d s}{s^{\frac{4}{3}}}[1-\cos (s \xi)] \sin ^{2}\left(s \tau^{\frac{3}{2}}-\frac{\pi}{12}\right) \\
& \Phi_{2}\left(\xi, \tau^{\frac{3}{2}}\right)=\int_{\frac{2}{3} \tau^{-\frac{3}{2}}}^{\frac{2}{3}} \frac{d s}{s^{\frac{2}{3}}}[1-\cos (s \xi)] \cos ^{2}\left(s \tau^{\frac{3}{2}}+\frac{\pi}{12}\right) .
\end{aligned}
$$

In the limit where $\tau \gg 1$ and $\xi \gg 1$, this expression can be further simplified and we find

$$
\mathfrak{H}(\xi, \tau) \simeq \frac{2^{\frac{4}{3}} \pi^{2} \tau^{\frac{1}{2}}}{K_{0} \Gamma\left(\frac{1}{3}\right)^{3}}\left(\xi^{\frac{1}{3}}-\frac{\Gamma\left(\frac{1}{3}\right)}{\pi}\left(\frac{3}{2}\right)^{\frac{1}{3}}\right)+\frac{2^{\frac{2}{3}} \pi \tau^{\frac{1}{2}}}{3 K_{0} \Gamma\left(\frac{2}{3}\right)^{2}}\left(2^{\frac{1}{3}} 3^{\frac{2}{3}}-\frac{\pi^{\frac{1}{2}} \Gamma\left(\frac{1}{6}\right)}{2^{\frac{2}{3}} \Gamma\left(\frac{1}{3}\right)} \frac{1}{\xi^{\frac{1}{3}}}\right),
$$

whereas in the limit where $\tau \gg 1$ and $\xi \ll 1$, we find

$$
\mathfrak{H}(\xi, \tau) \simeq 4 \pi \frac{\sqrt{\tau}}{K_{0}}\left(\frac{1}{53^{\frac{5}{3}} \Gamma\left(\frac{1}{3}\right)^{2}}+\frac{1}{73^{\frac{7}{3}} \Gamma\left(\frac{2}{3}\right)^{2}}\right) \xi^{2} .
$$

\section{Approximation for the full single-particle correlation function}

The full single-particle correlation function is given by $G(x, t)_{q \simeq 0}=A_{0}^{2} e^{-\frac{1}{2}(\mathfrak{G}(\xi, \tau)+\mathfrak{H}(\xi, \tau)+\mathfrak{Q}(\xi, \tau))}$. We now use the expressions derived above to find approximations for the entire function.

(i) Let us first discuss the limit of large distances such that $\xi \gg \tau^{\frac{3}{2}}$ (which necessarily implies that $\xi \gg 1$ ). In this limit, the large and intermediate momentum contributions become, to first order, independent of $\xi$ while the low momentum contribution $\mathfrak{G}(\xi, \tau) \simeq \frac{1}{K_{0}} \operatorname{Cin}\left(\frac{2 \xi}{3} \tau^{-\frac{3}{2}}\right) \simeq \frac{1}{K_{0}}\left(\gamma_{E}+\ln (\xi)\right)$ dominates. Thus the single-particle correlation behaves as

$$
G(\xi, \tau)_{q \simeq 0} \simeq \mathcal{A}(\tau)\left(\frac{1}{\xi}\right)^{\frac{1}{2 K_{0}}}
$$

where $\mathcal{A}(\tau)$ is a function which depends on $\tau$. We see that the Luttinger liquid behavior is recovered for long distances and that the exponent is the one expected in equilibrium before the ramp. 
(ii) Let us now discuss the case of small distances $\xi \ll \tau^{-\frac{1}{4}}$. In this limit, one obtains that the large momentum contribution, $\mathfrak{Q}(\xi, \tau)$, dominates since the intermediate and small momentum contribution vanish algebraically. Thus the correlation is well described by the expression

$$
G(\xi, \tau)_{q \simeq 0} \simeq \mathcal{B}(\tau)\left(1+\frac{\xi^{2}}{\tilde{\alpha}^{2}}\right)^{-\frac{\sqrt{\tau}}{4 K_{0}}}
$$

with $\mathcal{B}(\tau)$ a function depending on $\tau$.

(iii) For intermediate distances, finding an asymptotic expression for the single-particle correlation is a more subtle task. However, for $1 \ll \xi \ll \tau^{\frac{3}{2}}$, one obtains

$$
\begin{aligned}
G(\xi, \tau)_{q \simeq 0} \simeq \mathcal{C}(\tau) & \exp \left[-\frac{2^{\frac{1}{3}} \pi^{2} \tau^{\frac{1}{2}}}{K_{0} \Gamma\left(\frac{1}{3}\right)^{3}}\left(\xi^{\frac{1}{3}}-\frac{\Gamma\left(\frac{1}{3}\right)}{\pi}\left(\frac{3}{2}\right)^{\frac{1}{3}}\right)\right] \\
& \times \exp \left[-\frac{\pi \tau^{\frac{1}{2}}}{32^{\frac{1}{3}} K_{0} \Gamma\left(\frac{2}{3}\right)^{2}}\left(2^{\frac{1}{3}} 3^{\frac{2}{3}}-\frac{\pi^{\frac{1}{2}} \Gamma\left(\frac{1}{6}\right)}{2^{\frac{2}{3}} \Gamma\left(\frac{1}{3}\right)} \frac{1}{\xi^{\frac{1}{3}}}\right)\right] .
\end{aligned}
$$

where $\mathcal{C}(\tau)$ a function that depends on $\tau$. This expression is due to the contribution $\mathfrak{H}(\xi, \tau)$ at intermediate momentum, and for sufficiently large $\xi$ the first exponential is dominant.

\section{FOURIER TRANSFORM OF A STRETCHED EXPONENTIAL}

The Fourier transform of a stretched exponential function of the form $f(x)=e^{-x^{\beta}}$ with $\beta<1$ is given by the expression

$$
\mathcal{Q}(q)=\int_{0}^{\infty} d x e^{-x^{\beta}} e^{i q x}=\sum_{0}^{\infty} \int_{0}^{\infty} \frac{\left(-x^{\beta}\right)^{n}}{n !} e^{i q x}=\sum_{0}^{\infty} \frac{(-1)^{n}}{n !} \int_{0}^{\infty} d u \frac{u^{\beta n} e^{i u}}{q^{\beta n+1}}=\sum_{0}^{\infty} \frac{(-1)^{n}}{\Gamma(n+1)} \frac{\Gamma(\beta n+1)}{q^{\beta n+1}} e^{i \frac{\pi}{2}(\beta n+1)}
$$

which is a convergent sum. When $\beta=\frac{1}{3}$, the sum can be further evaluated by using properties of the $\Gamma$ functions, $\frac{\Gamma\left(\frac{n}{3}+1\right)}{\Gamma(n+1)}=$ $\frac{1}{3} \frac{\Gamma\left(\frac{n}{3}\right)}{\Gamma(n)}$, and definitions of the Hypergeometric functions [1], this exercise yields the result

$$
\mathcal{Q}(q)=\frac{e^{-i \frac{\pi}{2} \operatorname{sign}(q)}}{|q|}\left[1-\frac{\pi e^{-i \frac{\pi}{6} \operatorname{sign}(q)}}{(3|q|)^{\frac{1}{3}}} \mathrm{Hi}\left(\frac{-e^{-i \frac{\pi}{6} \operatorname{sign}(q)}}{(3|q|)^{\frac{1}{3}}}\right)\right]
$$

where $\mathrm{Hi}$ is the Scorer function [1-3].

[1] M. Abramowitz and I. Stegun, eds., Handbook of mathematical functions (Dover, New York, 1972).

[2] A. Gil, J. Segura, and N. M. Temme, Math. Comp. 70, 1183 (2001).

[3] A. I. Nikishov and V. I. Ritus, math-ph/0501062 (2005). 